\title{
Oxford-AstraZeneca COVID-19 Vaccine (AZD1222), an Approved, Non-Replicating Chimpanzee Adenovirus-Vectored Vaccine for the COVID-19 Pandemic
}

\author{
Chuchu Guo ${ }^{1}$
}

\begin{abstract}
The emergence of SARS-CoV-2 causes a severe pandemic since the year 2019. To meet the challenge, the rapid research and development on the vaccine are paid much attention. This article focuses on the design and development of a promising AZD1222 vaccine to prevent or reduce COVID-19. To clarify the mechanism of this vaccine, the virology of SARS-CoV-2 is also discussed: the properties, infectious mechanisms, interactions with human immune responses of SARS-CoV2 included. The antigens selected, platform, routes and regimes for this vaccine are explained. The ongoing clinical trials and results for AZD1222, development of combination use with another Russia's approved vaccine, and the distribution of AZD1222 itself are discussed.
\end{abstract}

Keywords: AZD1222, ChAdOx1 nCoV-19, Adenovirus Vector, SARS-CoV-2, COVID-19, Reviews.

${ }^{1}$ Guangdong Technion-Israel of Institute of Technology.

Article Info: Received: February 13, 2021. Revised: March 3, 2021.

Published online: March 10, 2021. 


\section{Introduction}

\subsection{SARS-CoV-2 Biology}

SARS-CoV-2, an RNA virus with an envelope and primarily respiratory pathogen, belongs to genus Betacoronavirus, family Coronaviridae, causing the COVID-19 outbreak, akin with SARS-CoV and MERS-CoV epidemic in 2003 and 2012, respectively. This virus with spherical or moderately pleomorphic morphology consists of four main structural proteins and RNA: spike (S) glycoprotein forming 'corona' structure with S1 (receptor-binding domain (RBD) included) and S2 subunit, transmembrane (M) glycoprotein, envelope (E) protein forming ring structure and nucleocapsid $(\mathrm{N})$ protein complexed with a single-stranded, positivesense RNA genome. (Ortiz-Prado et al., 2020). Although there is a high similarity between the RBD of SARS-CoV and SARS-CoV-2, several differences of amino acid sequences are found in the middle of the binding domain, optimizing the affinity of RBD to Angiotensin-converting enzyme 2 (ACE2). (Samavati \& Uhal, 2020, p. 2)

\subsection{SARS-CoV-2 Invasion}

A SARS-CoV-2 virus contacts mucus of the nose or mouth and travels through the respiratory tract, reaching bronchial and alveolar epithelial cells. The virus's RBD on the $\mathrm{S} 1$ subunit of spike protein, the most variable part of the virus, binds to the peptidase domain (PD) of ACE2 receptor protein on the surface of a host cell. S2 does not directly interact with the ACE2 receptor, but it provides functional elements essential for virions to fuse with the membrane. Host proteases such as cathepsins and TMPRSS4 cleave the carboxyl-terminus of the S1 subunit, which triggers the fusion machinery assisting the virus's entrance, (Samavati \& Uhal, 2020, p. 2) or called 'spike decapitation.' This receptor-mediated endocytosis is then realized by inserting a compressed residual part of spike stem, forming a funnellike structure on the host cell membrane, entrance of the virus's N protein, and RNA into the cell sequentially. From now on, it is the onset of replication. The virus utilizes host organelles, ribosomes, etc. included, to duplicate RNA, synthesize structural N, E, M and spike protein. In addition to those, protective vehicles against host defense are produced to wrap virus RNA, and others are used to assemble structural proteins. After assembling virions with RNA and structural proteins, new generation releases from the cell with the same machinery for entrance and poises to infect another cell.

\subsection{Human Immune Responses}

The innate immune system is the system that has non-specific immunity, including a first and second line of defense. This kind of immunity is inborn, produced by some anatomical barriers such as the skin, gastrointestinal tract, mucosa (the first barrier) and phagocytes with receptors activated by some signal molecules (second barrier) to exert protection via chewing up extraneous microorganisms and triggering inflammatory responses. Data suggests the SARS-CoV-2 virus could 
suppress activation of innate immune responses by inhibiting dendritic cells (DCs) and Interferons (IFNs) expression; therefore, a prolonged incubation or presymptomatic period could be explained. Besides, this dysfunction of the innate immune system incurs overexpression of inflammatory responses and immunopathology, embodied by the pronounced increase level of phagocytes such as neutrophils in the blood, which make COVID-19 are susceptible to seniors with immunosenescence. (Jeyanathan et al., 2020).

In contrast, the adaptive immune system is specific for an antigen (third line of defense), humoral immunity and cell-mediated immunity included, mainly carried out by lymphocytes. Once the infection happens, the viral protein will be detected by antigen-presenting cells, which could digest and present it on Class II major histocompatibility complex (MHC) then pass to and trigger $\mathrm{CD}^{+} \mathrm{T}$ cell for macrophage activation and antibody production; on Class I MHC, then pass to and trigger $\mathrm{CD}^{+} \mathrm{T}$ cell for clearance of antigen-expressing target cells. Adaptive immune responses also interact with SARS-CoV-2 in various ways. The neutralizing antibodies target the RBD of coronaviruses on S protein and block its interaction with ACE2. In contrast, other antibodies occupy other sites of S1 and S2 to defend the virus via dampening conformational change of $\mathrm{S}$ protein then forestall membrane fusion machinery. Antibodies against $\mathrm{N}$ protein are also generated: Immunoglobulin $\mathrm{G}$ subtype2a (IgG2a). Although these kinds of antibodies could not neutralize the SARS-CoV-2 directly, they may protect the host via Fc-mediated effector functions. Research reveals that $\mathrm{S}$ protein-specific $\operatorname{IgA}$ is more predominant and robust with an earlier peak of responses than those of IgM. However, the appropriate titer of neutralizing antibodies for treatment or prevention still needs to be assured. Studies suggest to exert adequate protection, T cell-mediated immunity is also indispensable. $\mathrm{CD} 4^{+} \mathrm{T}$ cells ( $\mathrm{T}$ helper $\left(\mathrm{T}_{\mathrm{H}}\right)$ cell) assist antibody responses and activate $\mathrm{CD} 8^{+} \mathrm{T}$ cells (cytotoxic $\mathrm{T}$ cell). The viral infection postpones the activation of $\mathrm{T}$ cells in addition to the innate immune system. In convalescent cases from mild COVID-19, strong T cell responses against $\mathrm{N}, \mathrm{M}, \mathrm{S}$ proteins were identified by IFN- $\gamma$ ELISPOT, while only N-specific T cell responses remained later. In all cases of ICU patients with moderate to severe ARDS caused by COVID19 , both $\mathrm{CD} 4^{+}$and $\mathrm{CD} 8^{+} \mathrm{T}$ cells were detected. In cases of individuals with a higher incidence of hCoV infections, such as children and younger patients, the crossreactivity of $\mathrm{CD} 4 \mathrm{~T}$ cells exists, which could strengthen immune responses to SARS-CoV-2. However, hyperinflammation is also possibly generated by $\mathrm{T}$ cell responses and exacerbate disease severity of COVID-19. (Jeyanathan et al., 2020).

\section{Vaccine Design}

AZD1222 (formerly ChAdOx1 nCoV-19), one of the leading candidates for COVID-19 vaccine development, is the most clinically advanced vaccine with a viral-vectored platform under phase III clinical trial, developed by the University of Oxford, UK, and AstraZeneca. (Folegatti et al., 2020). 


\subsection{Scientific Rationale and Immunological Consideration}

With prior experience of the ChAdOx1-MERS vaccine for MERS-CoV, and ChAdOx1-TB for tuberculosis, the chimpanzee adenovirus-vectored vaccine has been widely researched with a well-established track record for control of the infectious disease. In addition to safety and gene malleability assured, published data show its excellent ability to induce robust antibodies and $\mathrm{T}$ cell responses for only a single dose or two repeated doses of intramuscular injection without an adjuvant. Technological support for large-scale clinical-grade production and storage of these kinds of vaccines is already established. What is more, its natural tropism of many serotypes to the respiratory epithelium is amenable for respiratory mucosal vaccination, which is the route more effective to induce antibodies, T cells and macrophage-mediated immunity, although the intramuscular injection for AZD1222 is generally applied at present. Compared with other adenovirus-vectored vaccines, such as the human serotype 5 adenovirus (Ad5), humans show minute to no pre-existing antivector immunity for ChAd, which could solve this critical issue related to the efficacy of this platform. (van Riel \& de Wit, 2020).

\subsection{Selection of SARS-CoV-2 Antigens}

Although there are M, N, E, S four structural proteins present in the infectious virion, only antibodies bind to spike (S) protein could successfully neutralize SARS-CoV2. Therefore, the whole $S$ protein is chosen to be the immunogen in this case. To express this immunogen, AZD1222, a replication-defective simian adenovirus vector could encode full-length structural surface glycoprotein of SARS-CoV-2 with a tissue plasminogen activator leader sequence designed via bioengineering. For AZD1222, the coding sequence of S protein is codon-optimized. (Folegatti et al., 2020).

\subsection{Vaccine Platform}

Recombinant viral vectors are among the most common COVID-19 vaccine development platforms, ranking only lower than the protein subunit vaccine. This platform consists of a recombinant virus attenuated to low pathogenicity and could either be non-replicating or replicating. AZD1222 is a non-replicating vector since no new virus particles are formed due to deletion of E1 (and E3) gene, (Guo et al., 2018) and immunity only triggered by vectors initially enter cells to produce vaccine antigens compared to replicating vector. Because of this endogenous antigen production, both humoral and cellular immune responses are induced. (van Riel \& de Wit, 2020). The two main requirements for a successful immunization are antigens from the target pathogen and an infection signal. In this case, the antigen chosen is $\mathrm{S}$ proteins, as reason mentioned above, and the infection signal is the pathogen-associated molecular pattern (PAMP). Research suggests that the adenovirus vector precipitate innate immune responses through TRL (Toll-like receptor)-dependent mechanisms to recognize several PAMPs such as triacyl lipopeptides recognized by TRL1. (Hartman et al., 2008). What is more, T cell 
responses, as part of adaptive immunity, against $S$ protein encoded by Ad vector, are stimulated by the activation of innate immune via the release of inflammatory cytokines. Antigen-presenting cells such as dendric cells then present this immunogen on MHC-I and -II to activate $\mathrm{CD} 4^{+}$and $\mathrm{CD} 8^{+} \mathrm{T}$ cells. PAMPs that interact with TLRs also promote helper $\mathrm{T}_{\mathrm{H}} 1$ - cell responses that assist the development and proliferation of cytotoxic $\mathrm{CD}^{+} \mathrm{T}$ cells for SARS-CoV-2 clearance, while $\mathrm{T}_{\mathrm{H}} 2$-cell responses assist maturation and isotype switching of $\mathrm{B}$ cells. (Thacker et al., 2009).

\subsection{Vaccine Routes and Regimens}

The route for AZD1222 administration is intramuscular (IM) injection for safety consideration. IM injection could activate protective $\operatorname{IgG}$ antibodies at the respiratory mucosa, but the mucosal IgA antibodies or $\mathrm{T}_{\mathrm{RM}}$ cells in the lungs could not be induced effectively. (Jeyanathan et al., 2020). Therefore, a respiratory mucosal (RM) route that could solve this problem will be considered in the future. Although this route is not suitable for most vaccine platforms because of unsafe immune adjuvants and repeated delivery, potent AZD1222, providing adequate protection with only one or two doses, has the potential to be administrated via RM route (but still need to be demonstrated with adequate data from clinical trials).

A standard single dose of $5 \times 10^{10}$ viral particles (vp) was relatively higher, designed in the pandemic situation to activate adequate neutralizing antibodies with the maximal possibility. Since the elderly with immunosenescence or comorbidities are significantly affected by COVID-19, the design of an appropriate dose for them to prevent infection or reduce severity is of great importance. (Folegatti et al., 2020).

The regimens for AZD1222 are one-or two-dosing (prime-boost dosing). According to studies in phase I/II clinical trials, a single dose could generate neutralizing antibodies and induce $\mathrm{T}$ cells response in the majority (85\% and $90 \%$, respectively, $\mathrm{n}=1077$ ) without serious adverse events (SAEs) occurring. (Bar-Zeev \& Moss, 2020). However, several studies in animal models suggest that a prime-boost strategy could enhance immunogenicity, which is promising to solve the problem of low vaccine efficacy among elderly groups. (Graham et al., 2020; Silva-Cayetano et al., 2020). According to the first full results from interim analysis summed from phase III data, one regime was given primarily with a low dose injection $\left(2.2 \times 10^{10}\right.$ or $\left.2.5 \times 10^{10} \mathrm{vp}\right)$ and at least one month apart, with a full dose (standard dose) injection. This regime showed 90\% efficacy (LD/SD regimen, $\mathrm{n}=2741)$ compared with another dosing regimen given as two full doses $(62.1 \%$ efficacy, $\mathrm{n}=8895$ ), also at least one month apart between each dose. The average efficacy of the two regimens is $70.4 \%(n=11,636)$. Both of the booster-dosing regimens were shown to induce more robust immune responses than a single-dose administration.

Authorized by the MHRA, the UK regulatory authority, for the emergency use, the planned rollout of the AZD1222 is two-dosing administration (SD/SD), 4-12 weeks 
apart and 12 weeks is estimated to be the optimal period according to the efficacy and current shortage of vaccine supplies. (Voysey, Costa Clemens, et al., 2021).

\section{Vaccine Development}

\subsection{Ongoing Clinical Trials}

A phase I/II single-blind, randomized controlled trial in the UK (Identifier: NCT04526990, numbered as COV001) for AZD1222 vaccination was conducted compared to a licensed meningococcal conjugate vaccination (MenACWY) as control. One thousand seventy-seven healthy adult participants between the ages 18-55 were enrolled. Different groups received One-dose or two-dose schedules. The efficacy and safety of this vaccine were the primary outcomes evaluated in this trial. (University of Oxford, 2020b). In the AZD1222 group receiving the one-dose injection, antibodies against SARS-CoV-2 reached a maximal value by day 28 and kept a relatively high level until day 56.

In contrast, in another group receiving a booster, the titers kept rising and reached five times more than the former group on day 56. T cell responses were detected as early as day 7 and peaked at day 14 . However, there was no increased intensity shown in the prime-boost group compared with the prime group. The trial shows no serious adverse events occurred among AZD1222 groups, while mild local and systemic reactions were reduced by supplementary prophylactic paracetamol that also helps exclude vaccine-related interference symptoms from COVID-19 symptoms without vitiation of immunogenicity. In summary, a single dose of the AZD1222 vaccine had higher reactogenicity, although it was safe and tolerated. (Folegatti et al., 2020).

A phase II/III trial (NCT04400838, single-blind, MenACWY as control, numbered as COV002) was conducted among a larger group of 12,390 adult volunteers in the UK. In this trial, the elderly, adults who had previously administrated a ChAdOxvectored vaccine and HIV-positive adults were additionally included compared with the previous trial. In this trial, the single dose, LD/SD and SD/SD administrations were received by different groups, and the timing between two doses was at least 4 weeks and up to 12 weeks apart. Further investigation and verification of efficacy and safety of AZD1222 vaccine were again the primary outcome measures, while other outcome measures such as exploratory immunology, the difference between batches, mucosal immunity are also studied. (University of Oxford, 2020a, p. 3).

Another phase III trial (ISRCTN89951424, numbered as COV003) was conducted in Brazil among a group of 10,300 adults, including the elderly. All participants in experimental groups were offered two doses of the vaccine at each dose of $3.5-$ $6.5 \times 10^{10} \mathrm{vp}$ with administration up to 12 weeks apart. This trial was also singleblind, but the control group for the second dose was changed to saline compared with previous studies (control group for the first dose remained the same: MenACWY injection) that primarily studied the virologically confirmed symptomatic cases of COVID-19 throughout 12 months. 
(ISRCTN - ISRCTN89951424, n.d.).

An additional double-blind phase I/II trial was initiated in South African adults with and without HIV-infection (NCT04444674, COV005). 2130 participants were enrolled in total, and saline placebo was taken as the control group. Again, experimental groups were offered two doses of the vaccine at a dose of $3.5-6.5 \times 10^{10} \mathrm{vp}$ with the administration, but second dose injection was up to 4 weeks apart this time. The new attention paid for this study was assessment of the incidence of adverse events in HIV-negative and -positive adults and the cellular (and humoral) immunogenicity of HIV-positive groups after vaccination. (University of Oxford, 2020c).

According to the interim analysis of the AZD1222 vaccine, the safety is assessed and summed from data of four trials above (COV001, COV002, COV003 and COV005), and the efficacy is assessed by a prespecified global pooled analysis, combined with the data for two phase III trials: COV002 and COV003 $(n=11,636)$. The safety assessment across four studies showed no hospitalizations or severe COVID-19 in participants receiving vaccine from 21 days after the first dose, and SAEs occurred in 168 participants from both the AZD1222 group and control group $(<1 \%, \mathrm{n}=23,745)$. For the AZD1222 group, the SAEs mainly consisted of cases of pyrexia and transverse myelitis. In the efficacy assessment among a diverse cohort, geographical and ethnic consideration included, AZD1222 was demonstrated with the efficacy of $70.4 \%$. (Voysey, Clemens, et al., 2021).

As mentioned in the section $\mathbf{2 . 4}$, the overall efficacy was given by two different regimens of dosing, the one-dosing regimen (LD/SD, 90.0\%) and two-dosing regimen, the one-dosing regimen $(\mathrm{LD} / \mathrm{SD}, 90.0 \%, \mathrm{n}=2741)$ and two-dosing regimen (SD/SD, 62.1\%, $\mathrm{n}=8895)$, which resulted in some criticisms. An explanation suggested that this difference could be generated by the various sizes of groups of the samples mentioned. In other words, if the groups injected with the LD/SD regimen is expanded, the efficacy for this regimen will be likely to drop. (Callaway, 2020). Another explanation is that, according to one preprint with the Lancet by the Oxford Vaccine Group, the timing of the booster dose could affect the immunogenicity and efficacy of AZD1222. The higher efficacy is obtained with a longer interval, which was corresponding with the data shown in interim analysis: the efficacy of LD/SD regimen was obtained with average longer time part than that of SD/SD regimen. (Voysey, Clemens, et al., 2021). Besides, in an SD/SD group $(n=1170)$, the binding antibody responses induced with an interval of more than 12 weeks were also more than two-fold higher than that with an interval of fewer than 6 weeks (2.19 GMR). Although more data from samples need to be included to reduce the effect of variation of individual sources on the efficacy assessment, there is possibly an underlying biological mechanism since the immunological data show the same trend with efficacy. (Voysey, Costa Clemens, et al., 2021).

An ongoing phase III trial is being conducted among a larger group of estimated 30,000 volunteers in the US (NCT04516746) as a rule of double-blind, randomized, Placebo-controlled test. In this trial, about one-third of the volunteers are injected with $0.9 \%(\mathrm{n} / \mathrm{V})$ saline as a placebo comparator, while the experimental group 
receives two-dose vaccine injection. (AstraZeneca, 2021). The primary efficacy endpoint is the symptomatic illness, and the safety endpoint is the occurrence of adverse events (AEs). In this trial, the race and aged group diversity is expanded. About a quarter of elderly participants $(23.6 \%)$ and over half of participants with comorbidities (57.8\%) are enrolled to study the effect of AZD1222 comprehensively. The estimated primary completion date will be March, 2021. (AstraZeneca, 2021).

\subsection{Combination use with Sputnik V vaccine}

Gam-COVID-Vac (alternative name: Sputnik V) is a heterologous human recombinant adenovirus (rAd)-based vaccine consisting of adenovirus serotype 26 (Ad26) and Ad5, developed by the Gamaleya Research Institute, with the efficacy of $91.6 \%(n=16,501)$ and high tolerance according to its interim analysis of phase III. (Logunov et al., 2021). However, human Ad5 is derived from a human cold virus, so that the pre-existing immunity for it may reduce the efficacy of vaccine towards COVID-19. In contrast, AZD1222 circumvent this issue since the ChAd is not derived from human. AstraZeneca joined Russia and began the clinical trials (NCT04686773 and NCT04684446) on a combination use of AZD1222 and one of the components of Sputnik V, rAd26-S, to boost the efficacy of the coronavirus vaccine. ("AstraZeneca Joins Russia to Boost Coronavirus Vaccine," 2021).

The participants will receive two doses, AZD1222 $\left(5 \times 10^{10} \mathrm{vp}\right)$ on Day 1 followed by rAd26-S $\left(1.0 \times 10^{11} \mathrm{vp}\right)$ on Day 29 , or in reverse order, rAd26-S on Day 1 followed by AZD1222 on Day 29 to determine the safety and immunogenicity of this combined vaccine. The estimated study start date will begin in February and March 2021. (AstraZeneca, 2020; R-Pharm, 2021).

\subsection{Practical Distribution}

Demonstrated by cases of accelerated vaccine production and distribution pipelines in the 2013-2015 EBOV outbreak, one of the advantages of adenovirus vectors is that they can be rapidly made to GMP at a large scale and distributed and convenient for storage at $2-8{ }^{\circ} \mathrm{C}$ (normal refrigerated conditions) for more than 6 months. (Sharpe et al., 2020). With minute modifications, AZD1222 was manufactured according to current Good Manufacturing Practice (cGMP) by the Clinical BioManufacturing Facility and approved by the Medicines and Healthcare products Regulatory Agency (MHRA). (Folegatti et al., 2020). Detailed evaluation of local country needs, priorities, community engagement and trust, are required to realize impaired distribution of future COVID-19 vaccines. Global remedy via AZD1222 vaccine is underway, while specific local realities should be thoroughly taken into consideration. (Bar-Zeev \& Moss, 2020).

Currently, AstraZeneca reached agreements with many governments such as the UK, US, Germany and France, and multilateral organizations from them to produce, supply and distribute the vaccine globally. A conditional marketing authorization 
(CMA) for AZD1222 is granted by the European Commission for the emergency use with 400 million additional doses of AZD1222 distributed in Europe soon. (Third Safe and Effective Vaccine against COVID-19, n.d.).

\section{Conclusion}

Oxford-AstraZeneca COVID-19 vaccine (AZD1222) is a non-replicating chimpanzee adenovirus-vectored vaccine to prevent COVID-19 with an efficacy of $70.4 \%$ assessed among a large cohort, serving as the third safe and effective vaccine authorized by the European Commission. It is a promising remedy for the epidemic status while in the long term, the safety and efficacy still need to be optimized by a change of regimens or co-development with other vaccines that support by an additional amount of data from clinical trials.

\section{References}

[1] AstraZeneca. (2020). A Phase I/II Open-label Randomized Study in Adults to Determine the Safety and Immunogenicity of AZD1222, a Non-replicating ChAdOx1 Vector Vaccine, Given in Combination With rAd26-S, Recombinant Adenovirus Type 26 Component of Gam-COVID-Vac Vaccine, for the Prevention of COVID 19 (Clinical Trial Registration

No. NCT04684446). clinicaltrials.gov. https://clinicaltrials.gov/ct2/show/NCT04684446

[2] AstraZeneca. (2021). A Phase III Randomized, Double-blind, Placebocontrolled Multicenter Study in Adults to Determine the Safety, Efficacy, and Immunogenicity of AZD1222, a Non-replicating ChAdOx1 Vector Vaccine, for the Prevention of COVID-19 (Clinical Trial Registration No.

NCT04516746). clinicaltrials.gov.

https://clinicaltrials.gov/ct2/show/NCT04516746

[3] AstraZeneca joins Russia to boost coronavirus vaccine. (2021). Nature Biotechnology, 39(1), 11-11. https://doi.org/10.1038/s41587-020-00804-4

[4] Bar-Zeev, N., \& Moss, W. J. (2020). Encouraging results from phase 1/2 COVID-19 vaccine trials. The Lancet, 396(10249), 448-449. https://doi.org/10.1016/S0140-6736(20)31611-1

[5] Callaway, E. (2020). Why Oxford's positive COVID vaccine results are puzzling scientists. Nature, 588(7836),16-18. https://doi.org/10.1038/d41586020-03326-w

[6] Folegatti, P. M., Ewer, K. J., Aley, P. K., Angus, B., Becker, S., BelijRammerstorfer, S., Bellamy, D., Bibi, S., Bittaye, M., Clutterbuck, E. A., Dold, C., Faust, S. N., Finn, A., Flaxman, A. L., Hallis, B., Heath, P., Jenkin, D., Lazarus, R., Makinson, R., Yau, Y. (2020). Safety and immunogenicity of the ChAdOx1 nCoV-19 vaccine against SARS-CoV-2: A preliminary report of a phase 1/2, single-blind, randomised controlled trial. The Lancet, 396(10249), 467-478. https://doi.org/10.1016/S0140-6736(20)31604-4 
[7] Graham, S. P., McLean, R. K., Spencer, A. J., Belij-Rammerstorfer, S., Wright, D., Ulaszewska, M., Edwards, J. C., Hayes, J. W. P., Martini, V., Thakur, N., Conceicao, C., Dietrich, I., Shelton, H., Waters, R., Ludi, A., Wilsden, G., Browning, C., Bialy, D., Bhat, S., Lambe, T. (2020). Evaluation of the immunogenicity of prime-boost vaccination with the replication-deficient viral vectored COVID-19 vaccine candidate ChAdOx1 nCoV-19. Npj Vaccines, 5(1), 1-6. https://doi.org/10.1038/s41541-020-00221-3

[8] Guo, J., Mondal, M., \& Zhou, D. (2018). Development of novel vaccine vectors: Chimpanzee adenoviral vectors. Human Vaccines \& Immunotherapeutics, 14(7), 1679-1685. https://doi.org/10.1080/21645515.2017.1419108

[9] Hartman, Z. C., Appledorn, D. M., \& Amalfitano, A. (2008). Adenovirus vector induced innate immune responses: Impact upon efficacy and toxicity in gene therapy and vaccine applications. Virus Research, 132(1), 1-14. https://doi.org/10.1016/j.virusres.2007.10.005

[10] University of Oxford. A phase III study to investigate a vaccine against COVID-19. ISRCTN. doi:10.1186/ISRCTN89951424 (2020).

[11] Jeyanathan, M., Afkhami, S., Smaill, F., Miller, M. S., Lichty, B. D., \& Xing, Z. (2020). Immunological considerations for COVID-19 vaccine strategies.

Nature Reviews Immunology, 20(10), 615-632. https://doi.org/10.1038/s41577-020-00434-6

[12] Logunov, D. Y., Dolzhikova, I. V., Shcheblyakov, D. V., Tukhvatulin, A. I., Zubkova, O. V., Dzharullaeva, A. S., Kovyrshina, A. V., Lubenets, N. L., Grousova, D. M., Erokhova, A. S., Botikov, A. G., Izhaeva, F. M., Popova, O., Ozharovskaya, T. A., Esmagambetov, I. B., Favorskaya, I. A., Zrelkin, D. I., Voronina, D. V., Shcherbinin, D. N., Gintsburg, A. L. (2021). Safety and efficacy of an rAd26 and rAd5 vector-based heterologous prime-boost COVID-19 vaccine: An interim analysis of a randomised controlled phase 3 trial in Russia. The Lancet, 0(0). https://doi.org/10.1016/S01406736(21)00234-8

[13] Ortiz-Prado, E., Simbaña-Rivera, K., Gómez- Barreno, L., Rubio-Neira, M., Guaman, L. P., Kyriakidis, N. C., Muslin, C., Jaramillo, A. M. G., Barba-Ostria, C., Cevallos-Robalino, D., Sanches-SanMiguel, H., Unigarro, L., Zalakeviciute, R., Gadian, N., \& López-Cortés, A. (2020). Clinical, molecular, and epidemiological characterization of the SARS-CoV-2 virus and the Coronavirus Disease 2019 (COVID-19), a comprehensive literature review. Diagnostic Microbiology and Infectious Disease, 98(1), 115094. https://doi.org/10.1016/j.diagmicrobio.2020.115094

[14] R-Pharm. (2021). A Phase II Open-label Study in Adults to Determine the Safety and Immunogenicity of AZD1222, a Non-replicating ChAdOx1 Vector Vaccine, Given in Combination With rAd26-S, Recombinant Adenovirus Type 26 Component of Gam-COVID-Vac Vaccine, for the Prevention of COVID 19 (Clinical Trial Registration No. NCT04686773). clinicaltrials.gov. https://clinicaltrials.gov/ct2/show/NCT04686773 
[15] Samavati, L., \& Uhal, B. D. (2020). ACE2, Much More Than Just a Receptor for SARS-COV-2. Frontiers in Cellular and Infection Microbiology, 10. https://doi.org/10.3389/fcimb.2020.00317

[16] Sharpe, H. R., Gilbride, C., Allen, E., Belij-Rammerstorfer, S., Bissett, C., Ewer, K., \& Lambe, T. (2020). The early landscape of coronavirus disease 2019 vaccine development in the UK and rest of the world. Immunology, 160(3), 223-232. https://doi.org/10.1111/imm.13222

[17] Silva-Cayetano, A., Foster, W. S., Innocentin, S., Belij-Rammerstorfer, S., Spencer, A. J., Burton, O. T., Fra-Bidó, S., Lee, J. L., Thakur, N., Conceicao, C., Wright, D., Barett, J., Evans-Bailey, N., Noble, C., Bailey, D., Liston, A., Gilbert, S. C., Lambe, T., \& Linterman, M. A. (2020). A booster dose enhances immunogenicity of the COVID-19 vaccine candidate ChAdOx 1 nCoV-19 in aged mice. BioRxiv, 2020.10.27.357426. https://doi.org/10.1101/2020.10.27.357426

[18] Thacker, E. E., Timares, L., \& Matthews, Q. L. (2009). Strategies to overcome host immunity to adenovirus vectors in vaccine development. Expert Review of Vaccines, 8(6), 761-777. https://doi.org/10.1586/erv.09.29

[19] European Commission (2021, Janaury 29). European Commission authorises third safe and effective vaccine against COVID-19. Brussels: Office for Official Publications of the European Communities.

[20] University of Oxford. (2020a). A Phase 2/3 Study to Determine the Efficacy, Safety and Immunogenicity of the Candidate Coronavirus Disease (COVID19) Vaccine ChAdOx1 nCoV-19 (Clinical Trial Registration No. NCT04400838). clinicaltrials.gov. https://clinicaltrials.gov/ct2/show/NCT04400838

[21] University of Oxford. (2020b). A Phase I/II Study to Determine Efficacy, Safety and Immunogenicity of the Candidate Coronavirus Disease (COVID19) Vaccine ChAdOx 1 nCoV-19 in UK Healthy Adult Volunteers (Clinical Trial Registration No. NCT04324606). clinicaltrials.gov. https://clinicaltrials.gov/ct2/show/NCT04324606

[22] University of Oxford. (2020c). An Adaptive Phase I/II Randomized Placebocontrolled Trial to Determine Safety, Immunogenicity and Efficacy of Nonreplicating ChAdOx1 SARS-CoV-2 Vaccine in South African Adults Living Without HIV; and Safety and Immunogenicity in Adults Living With HIV (Clinical Trial Registration No. NCT04444674). clinicaltrials.gov. https://clinicaltrials.gov/ct2/show/NCT04444674

[23] van Riel, D., \& de Wit, E. (2020). Next-generation vaccine platforms for COVID-19. Nature Materials, 19(8), 810-812. https://doi.org/10.1038/s41563-020-0746-0

[24] Voysey, M., Clemens, S. A. C., Madhi, S. A., Weckx, L. Y., Folegatti, P. M., Aley, P. K., Angus, B., Baillie, V. L., Barnabas, S. L., Bhorat, Q. E., Bibi, S., Briner, C., Cicconi, P., Collins, A. M., Colin-Jones, R., Cutland, C. L., Darton, T. C., Dheda, K., Duncan, C. J. A., Zuidewind, P. (2021). Safety and efficacy of the ChAdOx1 nCoV-19 vaccine (AZD1222) against SARS-CoV-2: An 
interim analysis of four randomised controlled trials in Brazil, South Africa, and the UK. The Lancet, 397(10269), 99-111. https://doi.org/10.1016/S01406736(20)32661-1.

[25] Voysey, M., Costa Clemens, S. A., Madhi, S. A., Weckx, L. Y., Folegatti, P. M., Aley, P. K., Angus, B. J., Baillie, V., Barnabas, S. L., Bhorat, Q. E., Bibi, S., Briner, C., Cicconi, P., Clutterbuck, E., Collins, A. M., Cutland, C., Darton, T., Dheda, K., Douglas, A. D., Group, O. C. V. T. (2021). Single Dose Administration, And The Influence Of The Timing Of The Booster Dose On Immunogenicity and Efficacy Of ChAdOx1 nCoV-19 (AZD1222) Vaccine (SSRN Scholarly Paper ID 3777268). Social Science Research Network. https://papers.ssrn.com/abstract=3777268. 